\title{
SOLIDÃO COMO VARIÁVEL PREDITORA NA DEPRESSÃO EM ADULTOS
}

\author{
Sabrina Martins Barroso \\ Universidade Federal do Triângulo Mineiro \\ Makilim Nunes Baptista \\ Universidade São Francisco \\ Cristian Zanon \\ Universidade Federal do Rio Grande do Sul
}

\begin{abstract}
Resumo
Depressão é um transtorno multifatorial, de alta prevalência, considerada um problema de saúde pública mundial. Diversas são as variáveis implicadas no desenvolvimento e manutenção deste transtorno, como as psicológicas, sociais e biológicas. O objetivo desta pesquisa foi avaliar a solidão como fator preditivo na depressão em adultos, após controle de outros preditores tais como ansiedade, estresse, neuroticismo e variáveis clínicas. A amostra foi composta de 297 universitários de Minas Gerais, São Paulo e Rio de Janeiro. As escalas utilizadas foram a EBADEP-A, DASS-21, UCLA, MHI-5 e IGFP-5. Após regressão múltipla hierárquica, os resultados demonstraram que a saúde mental, neuroticismo, ansiedade e solidão foram os principais preditores, sendo que a solidão, após controle de todas as variáveis, acresceu $1 \%$ de variância isolada no modelo e demonstrou ser uma das variáveis mais importantes relacionadas à depressão. A avaliação clínica e intervenções devem levar em consideração esta variável na prática clínica.
\end{abstract}

Palavras-chave: distúrbios afetivos; estados emocionais; fatores psicossociais

\section{LONELINESS AS A PREDICTIVE VARIABLE IN ADULT DEPRESSION}

\begin{abstract}
Depression is a multifactorial and high prevalence disorder considered a global public health problem. There are several variables involved in the development and maintenance of this disorder, including psychological, social and biological ones. The objective of this research was to evaluate loneliness as a predictive factor in adult depression, after controlling other predictors such as anxiety, stress, neuroticism and clinical variables. The sample consisted of 297 university students from Minas Gerais, São Paulo and Rio de Janeiro. The scales used were EBADEP-A, DASS-21, UCLA, MHI-5 and IGFP-5. After multiple hierarchical regression, the results showed that mental health, neuroticism, anxiety and loneliness were the main predictors. Loneliness, after controlling for all variables, increased $1 \%$ of exclusive variance in the model and was one of the most relevant predictors of depression. Clinical evaluation and interventions should consider loneliness in clinical practice.
\end{abstract}

Keywords: affective disorders; emotional states; psychosocial factors 


\title{
SOLEDAD COMO VARIABLE PREDICTORA EN LA DEPRESIÓN EN ADULTOS
}

\begin{abstract}
Resumen
La depresión es un trastorno multifactorial, de alta prevalencia, considerado un problema de salud pública mundial. Diversas son las variables implicadas en el desarrollo y mantenimiento de este trastorno, entre ellos psicológicos, sociales y biológicos. El objetivo de esta investigación fue evaluar la soledad como factor predictivo en la depresión en adultos, tras el control de otros predictores tales como ansiedad, estrés, neuroticismo y variables clínicas. La muestra fue compuesta de 297 universitarios de Minas Gerais, São Paulo y Río de Janeiro. Las escalas utilizadas fueron la EBADEP-A, DASS-21, UCLA, MHI-5 e IGFP-5. Después de la regresión múltiple jerárquica, los resultados demostraron que la salud mental, el neuroticismo, la preocupación y la soledad eran los preditores principales, y la soledad, después del control de todas las variables, aumentó un $1 \%$ de varianza aislada en el modelo y demostró ser una de las variables más importantes relacionadas con la depresión. La evaluación clínica e intervenciones deben tener en cuenta esta variable en la práctica clínica.
\end{abstract}

Palabras clave: distúrbios afetivos; estados emocionales; factores psicossociais

\section{INTRODUÇÃO}

A depressão é uma patologia com elevada prevalência, variando entre 3 e $17 \%$ ao longo da vida e atingindo 350 milhões de pessoas no mundo (Habert et al., 2016; World Health Organization [WHO], 2012) e entre as pessoas com depressão severa, 15\% cometem suicídio (Moreira \& Bastos, 2015). A depressão maior se caracteriza por humor rebaixado persistente e perda de interesse em atividades prazerosas, gerando grande prejuízo funcional e social, anos de vida perdidos ou vividos com incapacidade, além de agravar os sintomas de outras doenças (Barros et al., 2017; Thorsen, Rugulies, Hjarsbech, \& Bjorner, 2013).

A depressão é uma patologia com causas multifatoriais, que incluem aspectos de personalidade, sociais e de relacionamentos interpessoais, não podendo ser atribuída a uma causa única (Hankin \& Abela, 2005; Jaremka, Lindgren, \& Kiecolt-Glaser, 2013). Entre os aspectos de personalidade, o neuroticismo tem sido o fator mais relacionado com o surgimento de problemas emocionais, como ansiedade e depressão (Freire et al., 2006; Petito et al., 2016; Zanon, Borsa, Bandeira, \& Hutz, 2012). Neuroticismo é um traço de personalidade que envolve preocupação, mau humor e nervosismo (Hayward, Taylor, Smoski, Steffens, \& Payne, 2013) e índices mais elevados de neuroticismo já se mostraram relacionados à depressão em estudos com diferentes populações (Hayward et al., 2013; Kim, Linton, Cho, \& Ha, 2016) e também com ruminação e ansiedade em outros (Zanon et al., 2012).

Aspectos do cotidiano, como o sedentarismo, consumo excessivo de álcool, tabagismo e piores hábitos alimentares também têm sido relacionados à depressão (Barros et al., 2017; Fone, Dunstan, John, \& Lloyd, 2007). Outro fator que mais recentemente foi associado à depressão é a solidão (Cacioppo, Hawkley, \& Thisted, 2010; van Winkel et al., 2017).

Solidão não é sinônimo de estar sem outras pessoas por perto. Ela pode ser entendida como uma discrepância sobre a qualidade e/ou quantidade de 
relacionamentos interpessoais que uma pessoa deseja e os que ela possui (van Winkel et al., 2017). Estudos que investigaram a solidão indicaram sua relação com expectativas negativas sobre relações, antecipação de rejeições afetivas e pior resposta a estímulos negativos, aludindo a solidão como um processo evolucionista (Cacioppo et al., 2014; Hawkley \& Cacioppo, 2010). Relações entre a solidão e o isolamento social também foram observadas, mas a influência da solidão na depressão se mostrou mais marcada do que sobre o isolamento (Matthews et al., 2016).

Jaremka et al. (2013) observaram a inter-relação entre depressão, solidão estresse e relacionamentos problemáticos. No estudo conduzido por van Winkel et al. (2017), sobre o papel da solidão na depressão, observou-se que a solidão permaneceu como fator associado ao surgimento da depressão mesmo quando controlados os sintomas depressivos pré-clínicos no nível de base. Beutel et al. (2017) identificaram que a solidão aumenta o risco para depressão, transtorno de ansiedade e ideação suicida. Além disso, Boivin e Hymel (1997) indicaram que a solidão age como mediadora do impacto e das consequências dos sintomas depressivos em crianças, fazendo com que a depressão tenha piores e mais prolongadas consequências em crianças mais solitárias. A influência da solidão e da depressão também tem sido demonstrada sobre processos inflamatórios e outras condições físicas (Mezuk et al., 2016).

No Brasil, a investigação da solidão é recente e sua influência na depressão ainda é pouco investigada (Azeredo \& Afonso, 2016; Vicente et al., 2014). Azevedo e Afonso (2016) investigaram a solidão em idosos, identificando $21 \%$ de solidão na amostra avaliada. O estudo de Vicente et al. (2014), que investigou idosos institucionalizados, indicou que aqueles com mais sintomas depressivos também mostraram maiores níveis de solidão, ansiedade, afeto negativo e menores índices de afetos positivos. Entretanto, não foram localizados estudos com jovens ou adultos que investigassem a influência da solidão sobre a depressão em contexto brasileiro.

Considerando o impacto da depressão e os achados internacionais sobre a influência da solidão para o surgimento do transtorno, o presente estudo visou avaliar o papel preditivo da solidão na depressão em adultos, quando controlados outros preditores conhecidos (ansiedade, estresse, neuroticismo e aspectos sociodemográficos) para o transtorno. Partiu-se da hipótese que mesmo diante de outros preditores, a solidão continua sendo um importante fator associado à depressão. 


\section{MÉTODO}

\section{Participantes}

Responderam aos instrumentos 297 estudantes universitários (70\% mulheres) dos estados de Minas Gerais, São Paulo e Rio de Janeiro, com idades variando entre 17 e 76 anos $(M=27,3$; $D P=18,8)$. Aproximadamente, $78 \%$ dos participantes eram solteiros, $19 \%$ casados e os demais $3 \%$ eram viúvos ou separados. A maior parte dos participantes indicou que nunca teve problema de saúde importante $(86,3 \%)$, que não fazia uso de qualquer medicação $(71,2 \%)$ e que nunca foi diagnosticado com depressão por um médico ou psicólogo $(81,3 \%)$.

Esta amostra é adequada para a análise de regressão múltipla prevista, já que ultrapassa o critério mínimo sugerido por Tabachnick e Fidell (2007) para este tipo de análise. A fórmula apresentada pelas autoras sugere: $\mathrm{N}>50+8 \mathrm{~m}$. Onde $\mathrm{N}$ indica o tamanho amostral e $\mathrm{m}$ indica o número de variáveis independentes incluídas no modelo (por exemplo, 11).

\section{Instrumentos}

Escala Baptista de Depressão (Versão Adulto) - EBADEP-A.

Construída por Baptista (2012), apresenta 45 itens divididos em duas frases por item, com respostas em formato Likert de quatro pontos ( 0 a 3), unidimensional, que avalia um número maior de descritores quando comparado a outras escalas tradicionais que avaliam sintomatologia depressiva. A EBADEP-A é baseada nos descritores dos principais manuais psiquiátricos (DSM e CID), além das teorias cognitiva e comportamental. $O$ instrumento mostrou boas qualidades psicométricas, com alfa de Cronbach de 0,95, sensibilidade de $77,5 \%$ e especificidade de $87,5 \%$.

Depression, Anxiety and Stress Scale (DASS-21).

Desenvolvida por Lovibond e Lovibond (2004) e validada para o Brasil por Vignola e Tucci (2014), a escala tem 21 itens, divididos em três subescalas que avaliam sintomas de depressão, ansiedade e estresse. As respostas são apresentadas em escala Likert de 4 pontos (de 0 "não se aplicou nada a mim" a 3 "aplicou-se na maior parte das vezes"). O instrumento mostrou boas qualidades psicométricas, com alfa de Cronbach entre 0,80 e 0,92. A correção é feita multiplicando os valores das subescalas por dois e utilizando os pontos de corte da DASS-42 (Lovibond \& Lovibond, 1995). Para a presente análise foram utilizadas as subescalas de ansiedade e estresse.

Escala Brasileira de Solidão UCLA (UCLA-BR).

Desenvolvida por Russell, Peplau, \& Cutrona (1980) e validada para o Brasil por Barroso, Midgett, Carvalho e Andrade (2016), contém 20 afirmações, 
com respostas em escala tipo Likert de quatro pontos, indo de 1 (nunca) a 4 (frequentemente). O instrumento mostrou consistência interna de 0,94 e boa capacidade de discriminação dos itens. A pontuação varia de zero a 60, sendo: 0 a 22 pontos indicativo de solidão mínima; 23 a 35 pontos, solidão leve; 36 a 47 pontos, solidão moderada; e 48 a 60 pontos, solidão intensa.

Inventário de Saúde Mental de 5 itens (MHI-5).

Subescala de cinco itens que integra o SF-36 e avalia aspectos de saúde mental (McHorney \& Ware, 1995; Ware, Snow, Kosinski, \& Gandek, 1993), adaptada para o Brasil por Damásio, Borsa e Koller (2014). Escores mais altos indicam melhores níveis de saúde mental. As análises mostraram instrumento unifatorial, explicando $64,58 \%$ da variância do construto.

Inventário dos Cinco Grandes Fatores da Personalidade (IGFP-5).

Criado por John, Donahue e Kentle (1991), adaptado e validado para o contexto brasileiro por Andrade (2008), avalia cinco dimensões de personalidade (Abertura, Conscienciosidade, Extroversão, Amabilidade e Neuroticismo). O instrumento é composto por 44 afirmações a que os respondentes devem indicar o nível de concordância por meio de escala tipo Likert de cinco pontos (1= Discordo totalmente até $5=$ Concordo totalmente). Mostrou validade de construto e fidedignidade variando entre 0,68 a 0,76. Para a presente análise considerouse apenas o índice de neuroticismo levantado no IGFP-5.

\section{Questionário de Identificação Sociodemográfico e Clínico.}

Utilizado para coletar dados pessoais (sexo, idade), diagnóstico prévio de depressão, presença de outro transtorno mental, histórico familiar de transtorno psiquiátrico, tratamento para depressão e tentativa de suicídio.

\section{Procedimentos}

As escalas foram aplicadas coletivamente em salas de até 40 estudantes, com três tipos de protocolos aleatórios de sequência dos instrumentos, com finalidade de controle de viés de fadiga nas respostas, sendo sempre o questionário de identificação sociodemográfico e clínico a primeira parte. 0 tempo de resposta dos protocolos foi em média de 40 minutos.

Análise de dados

Realizou-se análise de regressão múltipla hierárquica em três passos considerando os fatores identificados na literatura como preditores para depressão. Inicialmente, incluiu-se variáveis sócio demográficas e referentes a histórico de depressão (passo 1). Posteriormente, incluiu-se no modelo variáveis referentes a outras psicopatologias e neuroticismo (passo 2). Por fim, incluiu-se ao modelo a variável solidão (passo 3), para avaliar se a variável apresentava variância explicativa exclusiva sobre as demais variáveis já incluídas. 
Considerou-se a depressão como variável dependente, as demais variáveis como independentes e adotou-se significância de 5\%.

\section{RESULTADOS}

O modelo inicial (passo 1 ) indicou que as variáveis sociodemográficas e referentes ao histórico de depressão explicaram, aproximadamente, $10 \%$ da depressão. O acréscimo de outras psicopatologias e neuroticismo (passo 2) acrescentou mais $60 \%$ de predição exclusiva sobre a depressão, totalizando, aproximadamente, $70 \%$ de variância predita ( $10 \%$ do passo $1+60 \%$ do passo 2). Por fim, o acréscimo de solidão ao modelo (passo 3 ) acrescentou $1 \%$ de variância exclusiva para o total explicado. O modelo final (incluindo passo 1 , passo 2 e passo 3) mostrou quatro variáveis explicativas significativas (e.g., ansiedade, neuroticismo, indicativo de saúde mental e solidão), sendo que o total da variância da depressão explicada foi de $71 \%$. Os preditores mais fortes, que apresentaram maiores (em módulo) coeficientes de regressão padronizados $($ e.g., $\beta)$, do modelo foram o índice de saúde mental e o nível de solidão.

Tabela 1.

Resultados das análises de regressão linear para os fatores preditores da depressão.

\begin{tabular}{crcccc}
\hline Fatores Preditores & $\beta$ & $\mathrm{DP}$ & $\mathrm{t}$ & Valor-p & \\
\hline 1. Constante & $-2,35$ & 1,59 & $-1,48$ & 0,141 & \\
2. Sexo & $-0,29$ & 0,16 & $-1,81$ & 0,071 & $R^{2}=0,10$ \\
3. Histórico familiar depressão & $+0,32$ & 0,17 & $+1,90$ & 0,059 & $F(3,54)=$ \\
4. Tentativa de suicídio & & & & & 17,18 \\
5. Diagnóstico de depressão & $+0,64$ & 0,41 & $+1,53$ & 0,126 & $(p=0,003)$ \\
6. Outros transtornos & $+0,81$ & 0,45 & $+1,77$ & 0,078 & [DW =1,81] \\
7. Tratamento de depressão & $-0,02$ & 0,18 & $-0,14$ & 0,885 & \\
& $+0,37$ & 0,50 & $+0,74$ & 0,456 & \\
1. Constante & $-1,34$ & 1,04 & $-1,29$ & 0,199 & \\
2. Sexo & $-0,82$ & 0,09 & $-0,82$ & 0,410 & \\
3. Diagnóstico de depressão & $-0,09$ & 0,27 & $-0,33$ & 0,741 & $R^{2}=0,70$ \\
4. Tratamento de depressão & $+0,05$ & 0,29 & $+0,17$ & 0,860 & $F(32,39)=$ \\
5. Outros transtornos & $-0,07$ & 0,10 & $-0,69$ & 0,489 & 87,19 \\
6. Histórico familiar depressão & $+0,10$ & 0,10 & $+1,03$ & 0,305 & $(p<0,001)$ \\
7. Tentativa de suicídio & & & & & {$[\mathrm{DW}=1,81]$} \\
8. Estresse & $+0,16$ & 0,25 & $+0,66$ & 0,510 & \\
9. Ansiedade & 0,00 & 0,01 & 0,00 & 0,998 & \\
10. MHI & $+0,03$ & 0,01 & $+2,10$ & 0,037 & \\
11. Neuroticismo & $-0,45$ & 0,08 & $-5,25$ & 0,000 & \\
& $+0,09$ & 0,01 & $+5,22$ & 0,000 & \\
\hline
\end{tabular}




\begin{tabular}{crrrrr}
\hline 1. Constante & $-1,53$ & 1,02 & $-1,50$ & 0,136 & \\
2. Sexo & $-0,08$ & 0,09 & $-0,82$ & 0,410 & \\
3. Diagnóstico de depressão & $-0,02$ & 0,27 & $-0,10$ & 0,919 & \\
4. Tratamento para depressão & $+0,19$ & 0,29 & $+0,63$ & 0,525 & $R^{2}=0,71$ \\
5. Outros transtornos & & & & & $F(31,15)=$ \\
6. Histórico familiar depressão & $-0,09$ & 0,10 & $-0,90$ & 0,368 & 88,72 \\
7. Tentativa de suicídio & $+0,11$ & 0,10 & $+1,18$ & 0,237 & $(p=0,00)$ \\
8. Estresse & $+0,16$ & 0,24 & $+0,66$ & 0,507 & [DW =1,81] \\
9. Ansiedade & 0,00 & 0,01 & $-0,19$ & 0,848 & \\
10. MHI & $+0,04$ & 0,01 & $+2,25$ & 0,026 & \\
11. Neuroticismo & $-0,39$ & 0,08 & $-4,39$ & 0,000 & \\
12. Solidão & $+0,08$ & 0,01 & $+4,82$ & 0,000 & \\
& $+0,16$ & 0,06 & $+2,42$ & 0,017 & \\
& & & & & \\
\hline
\end{tabular}

\section{DISCUSSÃO}

Os resultados mostraram que diferentes fatores contribuíram para explicar o surgimento da depressão e que a solidão permaneceu como fator explicativo mesmo quando controlados outros aspectos. A existência de múltiplos preditores para a depressão é esperada, devido ao caráter complexo dessa patologia (Borges, Baptista, \& Serpa, 2017; Jaremka et al. 2013; Moreira \& Bastos, 2015). De forma semelhante, a presença de pior indicativo de saúde e ansiedade no modelo explicativo para a depressão corrobora com investigações anteriores sobre transtornos emocionais (Freire et al., 2007; Habert et al., 2016; Thorsen et al., 2013).

A presença de sintomas de humor, tais como sintomas ansiosos ou de pânico em conjunto com sintomas depressivos têm sido relacionados com vários transtornos psiquiátricos, mostrando a importância de considerar comorbidades ao tratar aspectos emocionais (Freire et al., 2006; Habert et al., 2016). De forma semelhante, a influência do nível de saúde mental já havia sido identificada em estudos anteriores (Fone et al., 2007; Thorsen et al., 2013). Ainda, construtos como a desesperança, desamparo, auto-eficácia, autoestima, autoconceito e solidão parecem estar relacionados com a depressão (Borges et al. 2017).

O neuroticismo também permaneceu no modelo explicativo. Como esse aspecto da personalidade está relacionado com pensamentos ruminativos e sentimentos mais negativos, sua relação com transtornos emocionais já havia sido identificada anteriormente (Zanon et al., 2012). No trabalho de Petito et al. (2016) o neuroticismo serviu como mediador da influência do gen $5 \mathrm{HTT}$ e a depressão. Kim et al. (2016) avaliaram preditores da depressão e identificaram que a idade, o sexo, renda, neuroticismo e desesperança se relacionavam com a depressão em imigrantes coreanos idosos. Entender que um aspecto de personalidade interfere como mediador de situações sociais e no surgimento de 
patologias emocionais aumenta a importância de pensar em intervenções multifatoriais, que considerem aspectos individuais e sociais.

Os resultados do presente estudo mostraram que a solidão é um importante fator preditor da depressão, mesmo que não responda por uma grande variância dos dados. Internacionalmente os estudos que consideraram o papel da solidão para o surgimento da depressão também mostraram a importância desse construto (Majd Ara, Telepasand, \& Razaei, 2017; van Winkel et al., 2017). No estudo de van Winkel et al. (2017) dois grupos foram acompanhados, sendo que um deles desenvolveu depressão e o outro não. No grupo que desenvolveu depressão, a solidão, a pior percepção dos relacionamentos sociais e passar mais tempo sozinho foram os fatores identificados como associados ao transtorno. Na investigação de Majd Ara et al. (2017) observou-se que a solidão foi um forte preditor para depressão e ainda atuou como mediador entre os relacionamentos sociais ruins e a depressão. Como a solidão ainda é foco de poucas investigações, a identificação de sua relação como preditora da depressão pode indicar um foco útil para prevenção da patologia. Intervenções voltadas para a solidão podem ajudar a minimizar a percepção de não estar emocionalmente conectado a ninguém, o que tem impacto em relacionamentos sociais, estratégias de enfrentamento, problemas físicos e emocionais.

A depressão é um transtorno com elevado custo para quem a possui e os resultados do presente estudo mostraram que a solidão pode ser um importante preditor para a depressão, que ainda tem sido pouco considerada. A influência da solidão, a forma como esse estado emocional interfere em aspectos físicos e emocionais, torna relevante considerá-la em investigações e intervenções futuras.

Como esse foi um estudo transversal não foi possível acompanhar mudanças no quadro depressivo ou identificar relações temporais entre a solidão e a depressão. Além disso, a amostra foi selecionada por conveniência, o que não permite generalização. Apesar de tais limitações, os dados permitiram identificar as relações de interesse. Ressalta-se que mais estudos sobre as relações de depressão e solidão são necessários para produção de evidências adicionais sobre as relações destas variáveis, principalmente de acompanhamento das variáveis de maneira longitudinal, em pacientes com quadro depressivo.

\section{CONSIDERAÇÕES FINAIS}

Considerando os resultados observados, intervenções focadas na redução dos níveis de neuroticismo e de solidão, podem ser desenvolvidas de formas individuais ou grupais, podendo auxiliar na prevenção ou minimizar sintomas depressivos. Especificamente para a população de estudantes universitários, 
pode-se pensar, ainda, em construção de espaços coletivos para socialização nas universidades e programas que ajudem na formação de vínculos com colegas universitários, reduzindo, com isso, a sensação de solidão e com potencial impacto na redução de sintomas ansiosos e depressivos.

\section{DECLARAÇÃO DE CONFLITO DE INTERESSES}

Dr. Makilim Nunes Baptista foi editor de seção para outros artigos do número especial que compõe esta edição da revista.

\section{REFERÊNCIAS}

Azeredo, Z. A. S., \& Afonso, M. A. N. (2016). Solidão na perspectiva do idoso. Revista Brasileira de Geriatria e Gerontologia, 19(2), 313-324. doi: 10.1590/1809-98232016019.150085

Baptista, M. N. (2012). Escala Baptista de Depressão (Versão Adulto) - EBDEP-A. São Paulo, SP: Vetor.

Barros, M. B. A., Lima, M. G., Azevedo, R. C. S., Medina, L. B. P., Lopes, C. S., Menezes, P. R., \& Malta, D. C. (2017). Depressão e comportamentos de saúde em adultos brasileiros - PNS 2013. Revista de Saúde Pública, 51, 1s10s. doi: 10.1590/S1518-8787.2017051000084

Barroso, S. M., Midgett, A. H., Carvalho, R. G. N., \& Andrade, V. S. (2016). Evidências de validade da Escala Brasileira de Solidão UCLA. Jornal Brasileiro de Psiquiatria, 65(1), 68-75. doi:10.1590/0047-2085000000105

Beutel, M. E., Klein, E. M., Brähler, E., Reiner, I., Jünger, C., Michal, M., Wiltink, J. ... Tibubos, A. N. (2017). Loneliness in the general population: Prevalence, determinants and relations to mental health. BMC Psychiatry, 17(97). doi:10.1186/s12888-017-1262-x

Boivin, M, \& Hymel S. (1997). Peer experiences and social self-perception: A sequential model. Developmental Psychology, 33(1), 135-145. doi: 10.1037//0012-1649.33.1.135

Borges, L., Baptista, M. N., \& Serpa, A. L. Oliveira. (2017). Structural analysis of depression indicators scale-children and adolescents (BAID-IJ): A bifactorESEM approach. Temas em Psicologia, 25(2), 545-552. doi: 10.9788/TP2017.2-08

Cacioppo, J. T., Cacioppo, S., \& Boomsma, D. I. (2014). Evolutionary mechanisms for loneliness. Cognition and Emotion, 28(1), 3-21. doi: 10.1080/02699931.2013.837379

Cacioppo, J. T., Hawkley, L. C., \& Thisted, R. A. (2010). Perceived social isolation makes me sad: 5-year cross-lagged analyses of loneliness and depressive symptomatology in the Chicago Health, Aging, and Social Relations Study. Psychology and Aging, 25(2), 453-463. doi:10.1037/a0017216 
Damásio, B. F., Borsa, J. C., \& Koller, S. H. (2014). Adaptation and psychometric properties of the brazilian version of the five-item Mental Health Index (MHI-5). Psicologia: Reflexão e Crítica, 27(2), 323-330. doi:10.1590/16787153.201427213

Fone, D. L., Dunstan, F., John, A., \& Lloyd, K. (2007). Associations between common mental disorders and the Mental Illness Needs Index in community settings. British Journal of Psychiatry, 191(2), 158-163. doi: 10.1192/bjp.bp.106.027458

Freire, R. C, Lopes, F. L., Veras, A. B., Valença, A. M., Mezzasalma, M. A., Nascimento, I. \& Nardi, A. E. (2007). Personality traits spectrum in panic disorder and major depression. Revista Brasileira de Psiquiatria, 29(1), 3134. doi: 10.1590/S1516-44462006005000009

Habert, J., Katzman, M. A., Oluboka, O. J., McIntyre, R. S., McIntosh, D., MacQueen, G. M., ... Kennedy, S. H. (2016). Functional recovery in Major Depressive Disorder: Focus on early optimized treatment. Primary Care Companion for CNS Disorders, 18(5), e1-e11. doi:10.4088/PCC.15r01926

Hankin, B. L., \& Abela, J. R. Z. (2005). Development of psychopathology: A vulnerability-stress perspective. Thousand Oaks: Sage Publications, Inc.

Hawkley, L. C., \& Cacioppo, J. T. (2010). Loneliness matters: A theoretical and empirical review of consequences and mechanisms. Annals of Behavioral Medicine, 40(2), 218-227. doi:10.1007/s12160-010-9210-8

Hayward, R. D., Taylor, W. D., Smoski, M. J., Steffens, D. C., Payne, M. E. (2013). Association of NEO personality domains and facets with presence, onset, and treatment outcomes of major depression among older adults. American Journal of Geriatric Psychiatry, 21(1), 88-96. doi: 10.1016/j.jagp.2012.11.012

Jaremka, L. M., Lindgren, M. E., \& Kiecolt-Glaser, J. K. (2013). Synergistic relationships among stress, depression and troubled relationships: Insights from psychoneuroimmunology. Depression and Anxiety, 30(4), 1-8. doi: $10.1002 /$ da. 22078

John, O. P., Donahue, E. M., \& Kentle, R. L. (1991). The Big Five Inventory Versions 4a and 54. Berkeley, CA: University of California, Berkeley, Institute of Personality and Social Research.

Kim, B. J., Linton, K., Cho, S., \& Ha, J. H. (2016) The Relationship between neuroticism, hopelessness, and depression in older Korean immigrants. PLOS ONE, 11(1), 1-10. doi:10.1371/journal.pone.0145520

Lovibond, S. H., \& Lovibond, P. F. (1995). Manual for the Depression Anxiety Stress Scales. 2nd ed., Psychology Foundation, Sydney.

Lovibond, S. H., \& Lovibond, P. F. (2004). Manual for the Depression Anxiety Stress Scales. 4th ed., Psychology Foundation, Sydney. 
Majd Ara, E., Telepasand, S., \& Razaei, A. M. (2017). A structural model of depression based on interpersonal relationships: The mediating role of coping strategies and loneliness. Archives of Neuropsychiatry, 54(2), 125130. doi: 10.5152/npa.2017.12711

Matthews, T., Danese, A., Wertz, J., Odgers, C. L., Ambler, A., Moffitt, T. E., \& Arseneault, L. (2016). Social isolation, loneliness, and depression in Young adulthood: A behavioural genetic analysis. Social Psychiatry and Psychiatric Epidemiology, 51(3), 339-348. doi:10.1007/s00127-016-1178-7

McHorney, C. A., \& Ware, J. E. (1995). Construction and validation of an alternate form of general mental health scale for the medical outcomes study short-form 36-item health survey. Medical Care, 33(1), 15-28.

Mezuk, B., Choi, M., DeSantis, A. S., Rapp, S. R., Roux, A. V. D., \& Seeman, T. (2016). Loneliness, depression and inflammation: Evidence of the multiethnic study of atherosclerosis. PLOS ONE, 11(7), 1-10. doi: $10.1371 /$ journal.pone.0158056

Moreira, L. C. O., \& Bastos, P. R. H. O. (2015). Prevalência e fatores associados à ideação suicida na adolescência: Revisão de literatura. Revista Quadrimestral da Associação Brasileira de Psicologia Escolar e Educacional, 19(3), 445-453. doi: 10.1519/2175-3539/2015/0193857

Petito, A., Altamura, M., Iuso, S., Padalino, F. A., Sessa, F., D'Andrea, G., ... Bellomo. A. (2016). The Relationship between personality traits, the 5HTT polymorphisms and the occurrence of anxiety and depressive symptoms in elite athletes. PLOS ONE, 11(6), 1-13. doi:10.1371/journal.pone.0156601

Russell, D., Peplau, L. A., \& Cutrona, C. E. (1980). The revised UCLA Loneliness Scale: concurrent and discriminant validity evidence. Journal of Personality and Social Psychology, 39(3), 472-80.

Tabachnick, B. G., \& Fidell, L. S. (2007). Using Multivariate Statistics (5th ed.). New York Allyn and Bacon.

Thorsen, S. V., Rugulies, R., Hjarsbech, P. U., \& Bjorner, J. B. (2013). The predictive value of mental health for long-term sickness absence: The Major Depression Inventory (MDI) and the Mental Health Inventory (MHI-5) compared. BMC Medical Research Methodology, 13(115), 1-7. doi: 10.1186/1471-2288-13-115.

van Winkel, M., Wichers, M., Collip, D., Jacobs, N., Derom, C., Thiery, E., ... Peeters, F. (2017). Unraveiling the role of loneliness in depression: The relationship between daily life experience and behavior. Psychiatry, 80(2), 104-117. doi:10.1080/00332747.2016.1256143

Vicente, F., Espirito-Santo, H., Cardoso, D., Silva, F., Costa, M., Martins, S., ... \& Lemos, L. (2014). Estudo longitudinal dos fatores associados à evolução de sintomas depressivos em idosos institucionalizados. Jornal Brasileiro de Psiquiatria, 63(4), 308-316. doi: 10.1590/0047-2085000000039 
Vignola, R. S. B., \& Tucci, A. M. (2014). Adaptation and validation of the Depression, Anxiety and Stress Scale (DASS) to Brazilian Portuguese. Research Report, 155, 104-109. doi:10.1016/j.jad.2013.10.031

Ware, J. E., Snow, K. K., Kosinski, M., \& Gandek, B. (1993). SF-36 health survey: Manual and interpretation guide. Boston: Medical Outcomes Trust.

WHO - World Health Organization [homepage internet]. Depression: A Global Public Health Concern. 2012; 6-8p. Recuperado de: http://www.who.int/mental_health/management/depression/who_paper_de pression_wfmh_2012.pdf.

Zanon, C., Borsa, J. C., Bandeira, D. R., \& Hutz, C. S. (2012). The relationship between ruminative thought and facets of neuroticism. Estudos de Psicologia, 29(2), 173-181. doi:10.1590/S0103-166X2012000200003

Sobre os autores

Sabrina Martins Barroso, psicóloga pela Universidade Federal de São João Del Rei, Mestre em Psicologia pela Universidade Federal de Minas Gerais, Doutora em Saúde Pública pela Universidade Federal de Minas Gerais. Professora adjunta da Universidade Federal do Triângulo Mineiro. Trabalha na linha de Avaliação Psicológica, Saúde Mental e Neuropsicologia. Recebe financiamento da Fundação de Amparo à Pesquisa de Minas Gerais. smb.uftm@gmail.com

Makilim Nunes Baptista é psicólogo pela Universidade São Judas Tadeu, mestre pela PUCCAMP e doutor pelo Departamento de Psiquiatria e Psicologia Médica da Escola Paulista de Medicina - UNIFESP Professor do Programa de Pós-Graduação Stricto-Sensu em Psicologia da Universidade São Francisco - Campinas - SP. Coordena o Laboratório de Avaliação Psicológica em Saúde Mental na mesma universidade (LAPSaM) e recebe financiamento de pesquisa pelo CNPq (Bolsa Produtividade). makilim01@gmail.com

Cristian Zanon é psicólogo pela Universidade Federal de Santa Maria, mestre e doutor em Psicologia pela Universidade Federal do Rio Grande do Sul. Trabalha nas linhas de Psicologia Positiva, Desenvolvimento Psicossocial e Psicometria e recebe financiamento de pesquisa do CNPq e FAPESP. crstn.zan@gmail.com

A contribuição de cada autor pode ser atribuída como se segue: S.M.B., M.N.B. e C.Z. contribuíram igualmente para a conceitualização, investigação e fizeram a redação inicial do artigo (rascunho) e a redação final (revisão e edição). Trabalharam na coleta de dados, análises, descrição, discussão e conclusões.

Recebido em: 04/09/2017

Revisado em: 20/04/2018

Aceito em: $16 / 06 / 2018$ 